
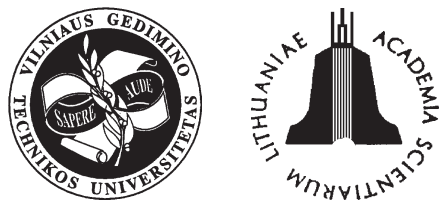

ISSN 1648-4142 TRANSPORT

http:/www.vtu.lt/english/editions

TRANSPORT-2005, Vol XX, No 1, 37-44

\title{
FUZZY INFERENCE DRIVEN INTERNET BASED BRIDGE MANAGEMENT SYSTEM
}

\author{
Deepesh Yadav, Sudhirkumar V. Barai \\ Dept of Civil Engineering Indian Institute of Technology Kharagpur, Kharagpur 721302 India. \\ E.mail:skbarai@civil.iitkgp.ernet.in.Phone:91-3222-283408,fax: 91-3222-282254
}

Received 2004-07-18; accepted 2004-12-03

\begin{abstract}
Bridges form an integral part of any country's transportation scheme, be it a railway network or a highway network. Periodic evaluation and subsequent maintenance can sometimes increase the life of a bridge to a great extent. Quantitative evaluation, however accurate, is too cumbersome, hence, in the present system the procedure of inspection is standardized and the analysis is done qualitatively. The qualitative data are converted to a mathematical format for the assessment by the use of popular Fuzzy logic approach. The whole system is based on the principle of evaluation from parts to the whole bridge structure. The method of calculation is based on Vertex method for fuzzy functions. The prototype system is the Internet based system and it can be accessed from anywhere as long as the area has the Internet access. The prototype system is an attempt in the direction of an organized and well-documented bridge management system.
\end{abstract}

Keywords: bridge management system, fuzzy logic, Internet, vertex method, visual inspection.

\section{Introduction}

The Internet is a set of interconnected computers that communicate with each other using the Internet Protocol (IP) and the Transmission Control Protocol (TCP). The World Wide Web $(W W W)$ is simply one application of the Internet. The Internet uses WWW to transmit multimedia documents written in a hypertext markup language (HTML) via the hypertext transfer protocol (HTTP) [1]. HTML is a markup language used to describe the format of multimedia documents and HTML documents are found using a browser. A browser running on one computer can request HTTP session from another browser. The two computers at either end of HTTP session play distinct roles. The client generates requests and receives responses. The server on the other hand receives requests and generates responses. Clients request HTML documents and servers provide them. With this description of Internet/WWW in mind, the following question can be addressed. Can the Internet/WWW be used to access and transfer the wide variety of information necessary in bridge management system (BMS)? The answer is yes.

As we know, bridges are the vital infrastructural element of Highway and Railway networks. There are approximately 1,11,500 bridges in service in Indian Railways [2]. These bridges range from small culverts of about $0.6 \mathrm{~m}$ span to $100-120 \mathrm{~m}$ multiple span bridges across important and mighty rivers. Most of the steel bridges in India are railway bridges. According to the published information [3], there are about 25000 steel bridges on the Indian Railways. These bridges can be classified mainly as plate girder type (upto $30,5 \mathrm{~m}$ span), open web type (30,5 $\mathrm{m}$ to $120 \mathrm{~m}$ span) and underslung (30,5 $\mathrm{m}$ to $45,7 \mathrm{~m}$ span). In addition there are some steel- concrete composite girders (upto 30,5 m span) too. Railway bridges are part of the nation's valued infrastructure. The older railway bridges were built around the turn of the century and their safety for transporting both freight and passenger trains is crucial. A significant number of old bridges [2] is distressed and many more get distressed every year during the service life under increased traffic. The increase of loads, the need for heavier traffic and the problem of fatigue necessitates the assessment of such bridge structures. This emphasizes the need of taking up a systematic and comprehensive investigation with reference to damage assessment of steel bridges in an integrated way [4].

In general bridge inspection covers visual inspection of each bridge component (e.g. deck, super-structure, sub-structure etc.) and subcomponents (e.g. joints, expansion bearing, main members etc.). Storing information in the form of photographs/ video films of the affected areas is an effective way of keeping permanent record of the condition of components/ subcomponents at the time of visual inspection. In order to capture the existing condition in its true per- 
spective the use of color polaroid camera has been recommended [5].

Usually pre-investigation exercise includes examining and checking the original calculations and drawings (if available), reviewing previous inspection repair records, and scheduling the bridge to be inspected taking into account the weather, stream levels, traffic volumes and bridge condition. Inspections and testing are conducted for the identified bridge components and subcomponents and the resulting data are analyzed for the determination of deficiencies. Results of these analyses are then summarized and interpreted by experienced engineers to yield final recommendations.

Maintaining records of serviceability of bridges and consequently retaining their level of reliability during their lifetime deserves high priority from techno-economic considerations. This aspect, unfortunately, has not hitherto been given the importance it deserves. For the optimal allocation of resources a clear cut procedure, guidelines and maintaining records are desirable which is the sole motivation underlining the development of BMS. BMS deals with recording information about the activities related to bridge right from their entry into service until their reconstruction. The key aspects addressed in a bridge management system are:

Inspection: Guidelines have been laid down by IRC [6] for inspection of road bridges covering culverts, minor and major bridges, as well as submersible bridges, bringing out what to look for during inspection of each class of bridge. The type of inspection has been classified together with their respective periodicity and level of engineering officers to inspect, depending on the importance and environment of bridge. The guidelines also indicate various inspection facilities necessary depending on the type and importance of the bridge and the tools and instrumentation to be used for inspection and testing.

Maintenance: It is defined as the work needed to preserve the intended load carrying capacity of the bridge to ensure the continued safety of users. It includes any work leading to the improvement of the structure whether by strengthening to carry heavier loads, by widening or vertical realignment of road surface. The scope maintenance operations start on the day the bridge becomes operational. The guidelines for maintenance cover aspects which can completely define the maintenance process.

Repair and Rehabilitation: The issue of remedies and rehabilitation is a separate entity and demands a highly empirical approach. Rehabilitation operations aim at restoring the bridge to the service level it once had and has now lost. In some cases this consists of giving the bridge service level which was intended, but which was never been attained because of the deficiencies in the original design and construction.

Here, in the presented paper, the aspects of inspection data utilization and the assessment are of key importance and deserve mathematical treatment. Various aspects of ratings of overall bridge structures have been discussed in the literature [5,7]. Comparative study was carried out using working stress, load factor and auto stress methods applied to the ratings of composite and non-composite steel beam girder bridges [8]. In order to improve the bridge condition rating procedure, Tee et al. $[9,10]$ used a Fuzzy mathematical approach.

The key issue to be addressed in the paper is to get an effective method of interpretation of the qualitative data using Fuzzy Logic and then mathematically manipulating it to yield reliable results [11]. The guidelines however are very general and will include only the elementary kind of bridges built under normal conditions. The bridges which are built in some special geography or those with special structural qualifications cannot be dealt directly and completely by the guidelines presented here.

Based on the above discussion the following objectives have been identified for the paper:

- Scheme identification of bridge structure subcomponents and components.

- Identification of linguistic terms for condition and important fuzzy variables (e.g. subcomponents and components).

- Identification of the suitable approach of fuzzy weighted average to combine linguistic conditions and importance of bridge components and subcomponents.

- Development of Graphics User Interface (GUI) and integrated system on platform independent platform - Internet Environment.

- Demonstration of the developed system.

Above the discussed objectives are explained in detail in the following sections. General discussion and limitations of the system are discussed before summarizing the paper.

\section{Scheme Identification for Bridge Structure Discretization}

The experimental evaluation of a bridge takes very long time and is not suitable for periodic inspection. Visual inspection can be one of the techniques which can help in inspecting bridges frequently. The inspector, if sees some abnormality, can recommend an experimental analysis for a particular structural component thereby reducing the overall work by eliminating redundant tests. Visual inspection of bridges 
is by nature a subjective process which does not lend itself to precise results. The inherent uncertainty in the inspection parameters together with the needs for intuition and judgment during inspection often resulted in the difficulties of maintaining consistency in bridge condition evaluation. Thus the use of inconsistent or weak bridge data may not be commensurate with the accuracy desired by rigorous bridge management systems.

As per the guidelines given by IRC [6] a bridge can be divided into 13 components for visual inspection. These 13 components can then be subdivided into subcomponents to make the evaluation process easy. The finer the discretization, the better will be our ability to judge. The discretization is done first into components and then these components are divided into parameters that can be used to judge them.

The overall scheme has these components:

- Approaches.

- Protective Work.

- Waterway.

- Foundation.

- Substructure.

- Bearings.

- Superstructure.

- Expansion Joints.

- Wearing Coat.

- Drainage Spouts and Vent Holes.

- Handrails and Parapets.

- Footpaths.

- Utilities.

These components further have been divided into subcomponents.

\section{(a) Approaches:}

- Condition of Pavement Surface (Settlement, Potholes, Cracking).

- Damage to side slopes.

- Damage to embankments by rain cuts etc.

- Condition of approach slab.

- Damage to retaining walls.

- Accumulation of silt and debris.

- Approach Geometrics.

\section{(b) Protective Work:}

- Damage to Layout.

- Condition of slope pitching, apron \& toe walls.

- Amount of scour.

- Quantity of Reserved store material.

- Condition of floor protection works.

(c) Waterway:
- Change in flow pattern.

- Maximum flood during the year.

- Afflux from upstream or downstream watermarks.

- Adequacy of waterway.

- Erosion of banks.

(d) Foundation:

- Settlement, scour and tilting.

- Disintegration, decay, erosion, cavitations etc.

- Damage due to impact of floating bodies, boulders etc.

- Damage to foundation.

- Seepage.

- Damage due to vehicular impact.

(e) Substructure:

- Efficiency of drainage of backfill behind abutments.

- Tilting, cracking, disintegration etc.

- Condition of side retaining walls.

- Excavation done in the road below in the vicinity of flyover or road over bridge of viaduct.

- Damage to protective measures to piers and abutments.

- Damage to protective coating or paint.

(f) Bearings:

- Metallic Bearings.

- General Condition (rusting, cleanliness etc.).

- Functioning.

- Greasing.

- Effectiveness of anchor bolts.

- Electrometric Bearings.

- Condition of pads.

- General cleanliness.

- Concrete Bearings.

- Distress.

- Shifting.

- Loss of shape.

- General cleanliness.

- Cracks in supporting members.

- Condition of downstream stoppers (for submersible bridges).

(g) Superstructure:

- Reinforced concrete and Prestressed concrete members.

- Spalling, disintegration, or honeycombing.

- Cracking.

- Exposed reinforcement. 
- Wear of deck surface.

- Scaling.

- Surface stains and rust stains.

- Leaching.

- Corrosion of reinforcement, sheathing \& tendons.

- Leakage.

- Damage due to moving vehicles.

- Condition of drainage.

- Condition of articulation.

- Excessive vibrations.

- Excessive deflections.

- Cracks around anchorage zone for prestressed members.

- Deflection at central hinge, (tip of cantilever for cantilever bridge).

- Cracking of interior faces of flanges and webs.

- Accumulation of water or debris.

- Cracking of interior diaphragms especially at their junctions to the webs.

- Accumulation of silt and debris on deck surface.

- Peeling off of protective coat or paint.

- Condition of steel members:

- Condition of Protective System.

- Corrosion.

- Vibrations.

- Alignment of members.

- Condition of connection (adequacy, looseness of rivets, bolts or worn-out welds, especially on connection of stringers to cross girders, cross girders to main girders, gussets or splices, condition of hinge spices etc.).

- Camber, deflections \& deformations.

- Buckling, kinking, warping \& waviness.

- Cleanliness of members \& joints (check choking of drainage holes provided in the bottom booms.)

- Fracture.

- Wears (in pins, in joints of truss) and their locations requiring close monitoring.

- Condition inside the closed members.

- Masonry Arches:

- Condition of joint mortar, pointing, masonry etc.

- Profile (report flattening by observing rise of the arch at the center and quarter points).

- Cracks ( indicate location, pattern, extent, depth).

- Drainage of spandrel fillings (check bulging of spandrel walls).

- Growth of Vegetation. (h) Expansion Joints:

- Functioning.

- Condition of sealing material.

- Secureness of joints.

- Damage to top sliding plate.

- Locking of joints.

- Debris in joints.

- Is there any rattling.

- Drainage for expansion joints.

- Alignment and clearance.

(i) Wearing Coat (Concrete and Bitumen):

- Surface Condition.

- Evidence of Wear.

- Design Thickness with respect to kerb height.

(j) Drainage Spouts and vent holes:

- $\quad$ Clogging, deterioration and damage.

- Protection of spout regarding adequacy.

- Drainage and pumping arrangements.

- Functioning.

(k) Handrails and Parapets:

- General Condition.

- Alignment.

\section{(1) Footpath:}

- General Condition.

- Cleanliness of ducts along footpaths.

(m) Utilities:

- Leakage of water and sewage pipes.

- Damage by electric and telephone cables.

- Condition of lightening facilities.

- Damage due to any other utilities.

These components are judged on the basis of some parameters relevant to them and the details of these parameters are given elsewhere [12].

\section{Identification of Linguistic Terms}

In general human beings are more used to express their opinions about visual inspection of bridge components and subcomponents in linguistic forms rather in numeric forms. This fact can be very well utilized while designing a system which is based on qualitative analysis. The proposed system tries to utilize this fact. The interpretation of linguistic data is by far an open issue and is available for any innovative treatment for its better interpretation. A branch of mathematics known as Fuzzy Logic attempts to resolve the ambiguity inherent in language processing. 
There are two parts of evaluation of components and subcomponents of bridges:

- Evaluate the importance/weightage of subcomponent based on its requirement in the overall functioning of the component.

- Evaluating its condition on the basis of its functioning.

The user has to express his opinion by making an appropriate selection from the choice list available. The choice list is prepared keeping in mind the specific requirements of a particular evaluation. For the purpose of providing a common choice list all the parameters are identified and classified on the basis of as to what is judged. The main categories in which the evaluation type is classified are: (i) Evaluating Importance (ii) Evaluating condition. (iii) Evaluating damage (iv) Checking whether functioning is satisfactory or not. (v) Checking of abnormalities.

The terms for various requirements are enumerated here:

\section{Fuzzy Variable Fuzzy Terms \\ 1 Condition Level Extremely Good, Very good,} Good, Medium, Poor, Very poor

2 Damage Level

Highly damaged, Significantly

damaged, Moderately, Less,

Slightly, Not damaged at all

3 Abnormalities Very high, High, Reasonable, Level Little, Very little, None

4 Functioning level Completely satisfactory, Quite satisfactory, Reasonably satisfactory, Barely satisfactory, Unsatisfactory, Highly unsatisfactory

\section{Importance Level Very Important, Important, Moderately Important, Less Important, Very less Important, Not Important}

Here since the procedure is visual inspection, our attempt is to make the inspection of very elementary components which can be easily and accurately judged. In this discretization scheme we have 3 or sometimes 4 layers. These subcomponents are further subdivided if they need further simplification. The inspection is done only of the subcomponent layer and the component layer conditions are computed.

\section{Fuzzy Weighted Average Approach}

Although the theory of fuzzy sets is relatively new, the calculus of fuzzy sets is well developed with various applications. For ready reference some basic fuzzy operations and interval arithmetic, required to compute a fuzzy function are given in [13].

Usually, from the experts' opinion surveys, various membership functions for component damage state and weightage are constructed and these are suitably translated into consistent fuzzy sets. The assignment of membership function of a fuzzy set is subjective in nature, and reflects the context in which the problem is viewed. It cannot be assigned arbitrarily.

The fuzzy sets are used as reference/standard sets for comparison. The mapping of fuzzy sets to natural language expression is a process of determining the distance from the resulting fuzzy set to each of the reference fuzzy sets representing a possible natural language expression. The mapping technique is illustrated elsewhere [14]. The governing expressions such as fuzzy Weighted Average which has been adopted for assessing the degree of global damage in bridge structures is illustrated in the next paragraph. The fuzzy Weighted Average in its simplest form is given as:

$$
y=\frac{\sum_{i=1}^{n} w_{i} x_{i}}{\sum_{i=1}^{n} w_{i}}
$$

$i=1,2, \ldots . ., n$ the number of members

Where, $x_{i}$ denotes the fuzzy condition rating of the $\mathrm{i}^{\text {th }}$ element and $w_{I}$ denotes the fuzzy importance factor for the same. $\left[w_{i a}, w_{i b}\right]$ and $\left[x_{i a}, x_{i b}\right]$ are $\alpha$-cut values of the fuzzy variable $w_{i}$ and $x_{i}$ corresponding to the fuzzy set $\mu_{w_{i a}}$ and $\mu_{x_{i a}}$. Once the fuzzy function $\mathrm{Y}$ (above expression) has been converted to the interval function $\mathrm{Y}$, the solution can be obtained using the Vertex method $[15,16]$. The above-discussed approach is used in the present study.

\section{System Development Process}

System development process involved:

- Implementation of the forms for various components and subcomponents of bridge structure.

- Implementation of the vertex method $[13,15,16]$.

- Integration of all the forms and the vertex method to get the unified system.

Forms here are the basic input device that we have. A form consists of the information as to how a component has to be inspected, it provides a user with 
an exhaustive option list from which he/she can select his opinion and finally forms are a means to store information for further processing. A typical form has the following features: (i) Graphical User Interface. (ii) The guidelines to inspect various components. (iii) An exhaustive list of options according to the kind of assessment. (iv) Means of acquiring and storing the data. All these features are implemented using Java [17] environment.

\section{Demonstration of the System}

The system developed using the above discussed concept is demonstrated with a typical user session [12]. Here due to space restriction only a few screen shots will be shown.

- After invoking the bridge management system in the Internet explorer environment a user will be welcome by a system. The welcome screen contains some information on the advantages of this system. Welcome screen provides the liberty to go to any page a user wishes.

- The next link is the theoretical basis which explains the basic concepts underlining the system mechanism.

- The next link is the overview (Fig 1). It explains the method of using this system. The help explains the users the way of expressing their opinions with the help of choice lists. Most of the things are intuitive here and follow naturally, thus no training is desired before using this system.

- The next page is the discretization scheme (Fig 2 ). The details have been discussed briefly in a previous section and in detail elsewhere [12]. The page gives an idea of the discretization scheme followed in the evaluation. It also gives an exhaustive list of parameters that are used to judge each component. The lists are implemented using collapsible lists economizing the space required. Clicking on a component displays the parameters used for judging it.

- The next link leads to the identification page (Fig 3 ). This page is the first step in the assessment of the bridge. It ascertains the identity of bridge and stores the data which are used to distinguish it from other bridges. This is done keeping in mind the extension of this system to include a database.

- The next form page holds the steering control of the whole assessment system. It contains the list of all the components (Fig 4) as given by IRC [6]. Any component can be selected by clicking the corresponding link and the system takes you to the inspection form of that component. One

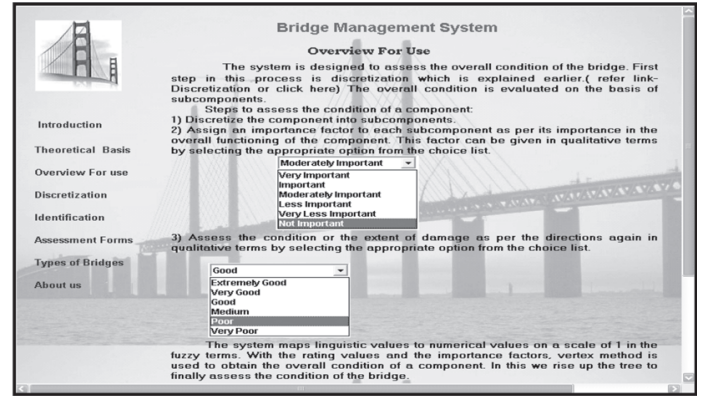

Fig 1. Overview page

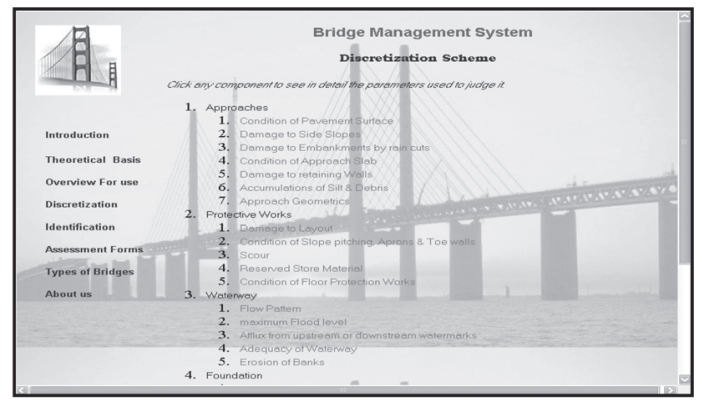

Fig 2. Discretization scheme

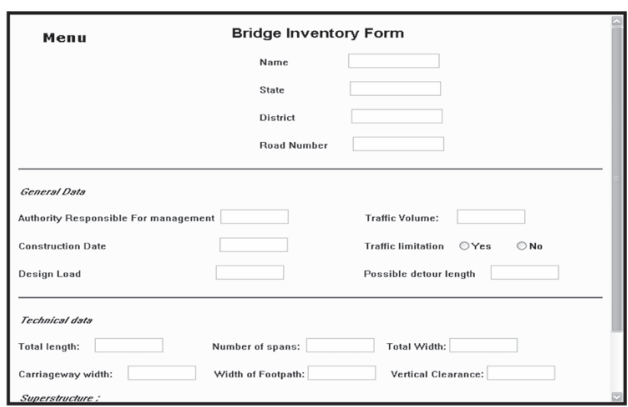

Fig 3. Inventory form

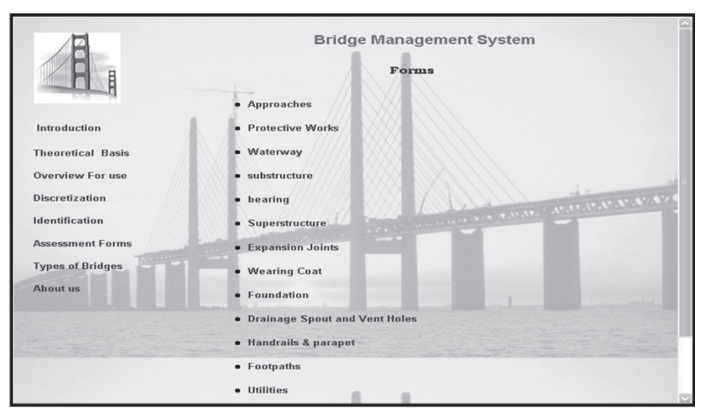

Fig 4. Forms page

example of component is given in Fig 5, which shows the assessment form of wearing coat. After reaching the form for a particular component, all one needs to do is to select appropriate options from the importance/weightage list and the rating list. After making proper selection press- 
ing submit will store the data and evaluate using Fuzzy Weighted Average and thereby giving the condition of that particular component. Similarly evaluation of each component is required to be done before going to the final evaluation page (Fig 6).

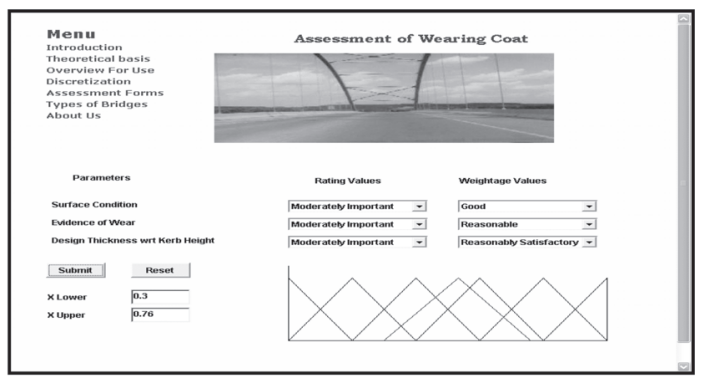

Fig 5. Assessment of wearing coat page

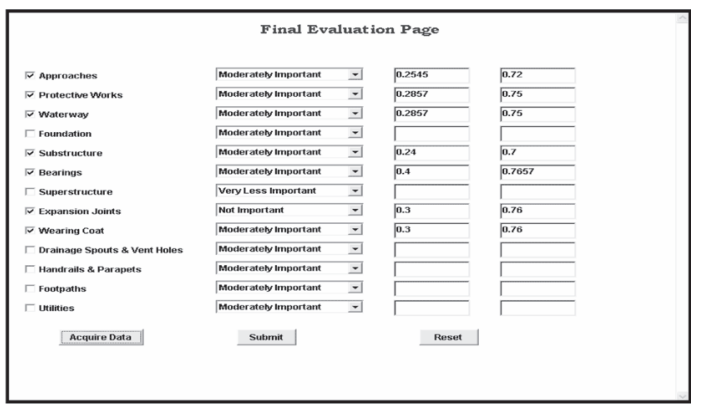

Fig 6. Final evaluation form

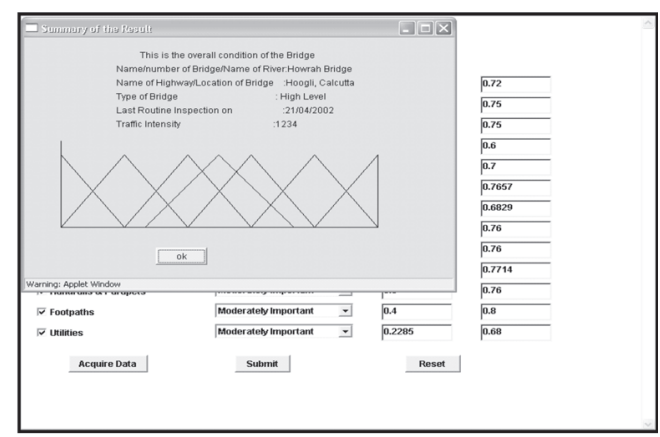

Fig 7. Final evaluation page showing the result

- Finally the whole comprehensive evaluation is done. The page is enabled with an error trapping mechanism so it is ensured that all the components are inspected before concluding the condition. The condition ratings for the components which have been computed can be acquired. Submitting these values will give an error message or if everything is done properly, a result window as in Fig 7 will appear after fuzzy weighted average computation. The blue function (triangle) corresponds to the result while the black ones are ref- erence functions. The result is in the form of a fuzzy function. It can be mapped back to the natural language expression for which various ranking algorithms are available. For the present paper gravity center criterion has been used [14].

- At last there are a few informative pages which carry general information about bridge and the other carries the contacts addresses of developers.

This completes the demonstration of the Bridge management system [12].

\section{General Discussion}

The following salient features of the bridge management system are identified:

- Global Coverage: With the continuing rapid spread of the web online system will be available to significant population of the globe: right from the novice to a competent bridge engineer or a bridge inspector.

- Interactive: This feature is actually the driving force behind the project. Every user has the option of interacting with the help of the interaction box provided in the applet. The user can very well manipulate the options and study the results to get a general idea as to how the system handles - Quick Result Generation: This package enables users to work out the overall condition of a complex structure in a very short time, once the data are acquired.

- User-friendly: The package has been made as userfriendly as possible to enhance the interaction with the user. Separate text-fields have been provided to guide the user during input. The help concerning the usage and methodology has been provided.

- Error trapping Mechanism: The system is enabled with an error trapping mechanism which ensures that every component is inspected before the final evaluation is done.

Limitations of Developed System:

- Bias Opinion: In the process of visual inspection there is always a possibility of 'biasness' from a bridge inspector. Handling biasness has been an open unresolved issue in literature. This limitation will always be there in any quantitative or qualitative evaluation of steel bridges.

- Discretization: For better applicability of this approach one can still divide the whole structure into smaller elements. The experienced inspectors can give the associated weightage factors to these components as crisp numbers. These weightage factors can be used to arrive at an over- 
all condition of a bridge for fuzzified condition rating of bridge components.

\section{Conclusion}

The choice of the Internet Based Bridge Management System stems from the wide accessibility that the Internet is capable of providing. Another reason to make this particular system web based is owing to the fact that the systems which are not available on the Internet are totally closed ended from the point of view of the user. The system has been developed using a fuzzy weighted approach. With advancement of Information Technology there is a scope to develop the system on wireless communication based Personal Digital Assistants (PDAs), Laptops and other mobile computers.

\section{References}

1. Schmuller, J. Dynamic HTML: Master the Essentials, BPB Publications, Connaught Place, New Delhi110001, 1998.

2. RDSO, Report of the sub-committee on rebuilding of railway bridges, Research Designs and Standards Organization, Govt. of India, Ministry of Railways, August, 1988.

3. Agrawal, S. R. Steel bridges in Indian Railways, in Advances in steel structures, V. Kalyanraman (editor). In: Proc. of the National Symposium on Advances in Steel Structures, Feb. 7-9, 1990, IIT Madras, Tata McGraw Hill, Publishing Company Ltd., New Delhi, 1990, 227 243.

4. Pandey, P. C. and Barai, S. V. Damage assessment of steel bridges. Journal of Structural Engineering (India), April, 1993, p. 9-12.

5. White, K. R.; Minor, J.; Derucher, K. N. and Heins, Jr. C. P. Bridge maintenance inspection and evaluation, Marcel Dekker Inc., 1981.

6. IRC-SP-35, Guidelines for Inspection and Maintenance of bridges. Indian Roads Congress Special Publication 35, New Delhi, 1990.

7. Koehn, E. and Barroeta, I., Inventory of highway infrastructure problems through bridge inspection. $A S C E$ Journal of Professional Issues in Engineering, Vol 117, No 2, 1991, p. 133-149.

8. Schelling, D. R. and Fu, C. C. Comparison of bridge rating methods. ASCE Journal of Structural Engineering, Vol 110, No 7, 1984, p. 1174-1179.

9. Tee, A. B.; Bowman, M. D.; Sinha, K. C. A Fuzzy mathematical approach for bridge condition evaluation. Civil Engineering Systems, Vol 5, March, 1988, p. 17-24.

10. Tee, A. B.; Bowman, and M. D.; Sinha, K. C. The development of optimal strategies for maintenance, replacement and placement of highway bridges. Final Report,
Vol 2, A system for bridge structural condition assessment, Joint Highway Research Project, FHWA/IN/ JHRP-89/9, Purdue University, 1990.

11. Folger, T. A. and Klir, G. J. Fuzzy Sets, Uncertainty and Information, Prentice hall of India Private Limited, New Delhi - 110001, 1991.

12. Yadav, D. Development of Internet Based Fuzzy Inference Driven Bridge Management System, B. Tech Thesis Report, Department of Civil Engineering, IIT Kharagpur, India, 2002.

13. Barai, S. V. and Pandey, P. C. Numerical Evaluation of Interval Based Fuzzy Functions (In the context of Expert Systems Development), Innovative Applications in Computing, Indian Computing Congress Series, Tata McGraw-Hill Publishing Company Limited, New Delhi. 1992.

14. Barai, S. V. Fuzzy Inference Based Procedure for Visual Inspection of Steel Bridges. IRC Journal, 61:3, 2000, p. 525-545.

15. Dong, W. M. and Shah, H. C., Vertex method for computing functions of fuzzy variables. Journal of Fuzzy Sets and Systems, Vol 24, 1987, p. 65-78.

16. Dong, W. M.; Shah, H. C. and Wong, F. S. Condensation of the knowledge base in expert systems with applications to seismic risk evaluation, in Expert systems in construction and structural engineering, Adeli, $\mathrm{H}$. (Editor), Chapman and Hall, London. 1988.

17. Naughton, P. and Schildt, H. Java 2: The Complete reference, Third edition, Tata McGraw-Hill Publishing Company Limited, New Delhi, 2000. 